\title{
Dual-polarization, wideband microstrip antenna array for airborne C-band SAR
}

\section{Granholm, Johan; Skou, Niels}

\section{Published in:}

Proceedings of Phased Array Systems and Technology

Link to article, DOI:

10.1109/PAST.2000.858949

Publication date:

2000

Document Version

Publisher's PDF, also known as Version of record

Link back to DTU Orbit

\section{Citation (APA):}

Granholm, J., \& Skou, N. (2000). Dual-polarization, wideband microstrip antenna array for airborne C-band SAR. In Proceedings of Phased Array Systems and Technology (pp. 243-246). IEEE.

https://doi.org/10.1109/PAST.2000.858949

\section{General rights}

Copyright and moral rights for the publications made accessible in the public portal are retained by the authors and/or other copyright owners and it is a condition of accessing publications that users recognise and abide by the legal requirements associated with these rights.

- Users may download and print one copy of any publication from the public portal for the purpose of private study or research.

- You may not further distribute the material or use it for any profit-making activity or commercial gain

- You may freely distribute the URL identifying the publication in the public portal

If you believe that this document breaches copyright please contact us providing details, and we will remove access to the work immediately and investigate your claim. 


\title{
DUAL-POLARIZATION, WIDEBAND MICROSTRIP ANTENNA ARRAY FOR AIRBORNE C-BAND SAR
}

\author{
Johan Granholm \& Niels Skou
}

Danish Center for Remote Sensing, Department of Electromagnetic Systems, Technical University of Denmark, Building 348, DK-2800 Lyngby, Denmark (jgeemi.dtu.dk, ns@emi.dtu.dk)

\begin{abstract}
The paper describes the development of a $\mathrm{C}$-band, duallinear polarization wideband antenna array, for use in the next-generation of the Danish airborne polarimetric synthetic aperture radar (SAR) system. The array is made of probe-fed, stacked microstrip patches. The design and performance of the basic stacked patch element, operating from $4.9 \mathrm{GHz}$ to $5.7 \mathrm{GHz}$, and a $2 \times 2$ element test-array of these, are described.

\section{Introduction}

High-resolution airborne and spaceborne imaging of the Earth is often carried out using remote sensing techniques, such as SAR. Early SAR systems were single-polarization instruments, but time has seen a growing interest in dualpolarization (i.e. "polarimetric") SAR systems. The reason for this trend is the additional amount of geophysical information, which it is possible to extract from polarimetric SAR data, compared to single-polarization data. It is well known, that radar signatures of e.g. crops are polarization dependent. An intuitive physical explanation of this dependence is, that the vertical polarization primarily are reflected by the vertical structures (e.g. straws, trunks), whereas the horizontal polarization are in stead reflected by the predominantly horizontal structures (e.g. branches). Mapping an area with polarimetric SAR thus provides more information, hence allows more details to be distinguished and increases the ability to classify targets. Future SAR systems for e.g. crop study and monitoring are therefore required to be polarimetric instruments. Several space agencies and other institutions are currently developing such next-generation polarimetric SAR systems, [1] - [4].
\end{abstract}

\section{Resolution requirements for future SAR systems}

Several polarimetric SAR systems have already been built and flown, incl. the Danish L- and C-band system, EMISAR developed at Department of Electromagnetic Systems, Technical University of Denmark (EMI), [5]. The EMISAR system has a resolution of approx. $2 \mathrm{~m}$ in both range and elevation. At the time of design (1993) this was state-of-the-art, but not least due to the rapidly advancing digital technology (especially high-speed data acquisition and storage systems), higher resolutions are possible today. At the same time user demands, e.g. for surveying applications, continue to call for increased resolution. Both for scientific and commercial mapping purposes, there are a desire to achieve resolution of $0.25 \times 0.25 \mathrm{~m}$.

The maximum SAR azimuth resolution is not dependent on the bandwidth of the transmitted radar chirp, but on the pulse-repetition frequency and to a first approximation to the physical antenna length, $l_{*}$. The SAR azimuthresolution, $\delta R_{A}$ is limited to:

$$
\delta R_{A}=l_{A} / 2
$$

This (frequency independent) result shows, that to obtain an azimuth resolution of $0.25 \mathrm{~m}$ the antenna should be max. 0.5 $m$ long. Lower frequency results in less gain with this limited antenna length. High-resolution SAR systems therefore are difficult at lower microwave frequencies.

The maximum range-resolution in SAR systems, $\delta R_{R}$, and the bandwidth requirement are inversely proportional:

$$
\delta R_{R}=c /\left(2 B_{R}\right)
$$

where $B_{\mathrm{R}}$ is the bandwidth of the transmitted pulse and $c$ is the speed of light. The present EMISAR system has 100 MHz bandwidth in both L-band and C-band, translating to approx. $2 \mathrm{~m}$ resolution in range, including proper weighting. To obtain $0.25 \mathrm{~m}$ resolution in range, the pulsed chirp must therefore have an eightfold increase in bandwidth. Although the range resolution of both the L-band SAR (with centre frequency $1.25 \mathrm{GHz}$ ) and the C-band SAR (centered at 5.3 $\mathrm{GHz}$ ) is desired to be increased in the next-generation EMISAR system, technology will not allow for this in Lband. In C-band, however, it may be possible to achieve up to $800 \mathrm{MHz}$ bandwidth. The goal therefore is to upgrade the, today medium resolution, C-band EMISAR to a highresolution system, capable of $800 \mathrm{MHz}$ operation. To be compatible with existing and planned civilian and scientific air- and spaceborne SAR's (to allow for data comparison), the centre frequency shall remain $5.3 \mathrm{GHz}$.

\section{Requirements for polarimetric SAR antenna arrays}

Future polarimetric SAR-systems require compact and lightweight dual-linear polarization antenna arrays, which should preferably be flat to facilitate easy installation; e.g. conformal on the fuselage of aircrafts. The arrays' elevation plane radiation patterns shall be shaped to resemble a modified cosecant-squared shape (to compensate for the range-dependence), while the azimuth plane radiation patterns shall be narrow, with a moderately low sidelobe level, and symmetrical w.r.t. boresight. In order to obtain a high degree of polarization discrimination of the overall SAR system, it is a further requirement to the array, that it should have a low cross-polarization level. An antenna element, which complies with these requirements, is the microstrip patch. Although it is very narrowbanded as a single-layer structure, a stacked patch can be added to significantly increase the bandwidth.

Aperture coupled microstrip patches, although capable of offering wide bandwidth, has the drawback that, in a practical antenna, a closed cavity is required to be placed behind the aperture. Such cavities may lead to a reduction of the bandwidth of the "naked" element but, worse, the cavity will take up valuable board space undemeath the aperture. Due to this "real-estate" problem, this cavity may prevent, or at least severely complicate, the (already challenging) design and layout of the necessarily multilayer beamforming network (BFN), which must reside under the groundplane, below the aperture. Furthermore, apertures are known to excite surface waves far stronger, than probe-fed patches. For these reasons aperture-coupled patches is not the optimum choice in this application. Probe-fed patches are attractive from the a feeding network point of view, since the probes does not take up any board space, and easily connect the patch to its beamforming network, which is e.g. located several layers down from the patch groundplane. For this reasons probe-fed patches are chosen.

The existing C-band EMISAR system [6], operating over an approx. $2 \%$ bandwidth, uses a single, probe-fed patch. The L-band EMISAR system [7], which is also operating over a $100 \mathrm{MHz}$ bandwidth (translating to $8 \%$ ), uses a stacked probe-fed patch. The idea thus was to investigate, if the stacked patch concept could be adopted and optimized to operate over an $800 \mathrm{MHz}$ bandwidth in C-band.

\section{Selection of dielectric substrate}

The bandwidth of microstrip patches depends strongly on the substrate thickness and -permittivity. For practical reasons, C-band patch probes shall be fabricated as "integrated via's". Although electrical attractive, this mechanical requirement preclude the use of low loss, low permittivity foam materials, since via's can neither be grown, nor supported, by such soft materials. Hence, for bandwidth reasons, it is desirably to use a thick, low 
permittivity hard substrate. On the other hand, the maximum thickness is limited for several reasons: A thick substrate may support the (undesirable) propagation of excessive surface waves, and will also be heavy. Furthermore, it will necessarily imply a long feeding probe, which electrically act as a large series-inductance, thus reducing the element bandwidth.

Due to fabrication and reliability issues, the multi-layer stripline $\mathrm{BFN}$ and the radiating patch layer should preferably be fabricated using the same type of substrate. The wide bandwidth requirement will demand that the elements must be fed through a binary-type network in the azimuth direction. In the elevation direction, it is desired to shape the elevation pattern, so that it will resemble a modified cosecant-squared pattern. This elevation BFN will be implemented using couplers and lines. It is desirable to be able to vary the characteristic impedance of the striplines over a wide range (w.r.t. $50 \Omega$ ), within acceptable linewidths (e.g. $5 \mathrm{~mm}$ to $0.5 \mathrm{~mm}$ ) to facilitate the design of these couplers, lines and necessary impedance matching networks. This requirement also calls for the use of a low permittivity substrate, although its thickness can be chosen independently of the patch substrate thickness. The design of the BFN, however, is a separate task, and will not be covered in this paper.

In this work, $32 \mathrm{mil}(0.8128 \mathrm{~mm})$ Rogers RO4003 substrate was initially chosen for the driven patch, having a dielectric constant $\varepsilon=3.38$ and loss tangent $\tan \delta=0.0027 @ 10 \mathrm{GHz}$ The $\mathrm{RO} 4003$ material is constructed as woven glass cloth, impregnated with a ceramic loaded thermoset plastic resin to yield a thermally stable rigid laminate with electrical properties suitable for microwave frequencies Although the RO4003 permittivity and dielectric loss factor is somewhat higher than PTFE-based materials (e.g. Rogers RT/duroid 5870 having $\varepsilon_{\mathrm{r}}=2.22$ and $\tan \delta=0.0009$ ( $10 \mathrm{GHz}$ ), RO4003 is attractive in an antenna feasibility study like the present, since RO4003 resembles FR4 in mechanical integrity and can be fabricated like basic FR4 material (and also because of its much lower price). Hence prototype development of stripline PCB's with integrated via's is much faster and cheaper if using RO4003 materials, than if using a PTFEmaterial.

Design of the $\mathbf{C}$-band wideband element

The starting point in the design of a stacked patch, is the design of the driven (i.e. single-layer) patch, shown in Fig. 1. It is designed so that its resonance frequency is in the centre of the band, i.e. $5.3 \mathrm{GHz}$



Fig.1. Dual polarization probe-fed microstrip single patch;

a) Top view of the patch, b) cross section.

The patch is fed close to its edges using $0.6 \mathrm{~mm}$ diameter probes (in the laboratory patch, the probe is directly made from the center conductor of a SMA-connector). Two probes (designated " $\mathrm{H}$ " and " $\mathrm{V}$ "), located on the patch" center lines, are used to excite the patch in orthogonal modes. This orthogonality will have the effect, that the coupling between the $\mathrm{H}$ and $\mathrm{V}$-ports (i.e. the scattering parameter $S_{11}$ ) remains fairly low in the vicinity of the patch resonance frequencies " "resonance frequency" is defined as the frequency at which the real part of the patchs' input impedance achieves its maximum value, when the reference plane is at the upper side of the ground plane). The measured input impedance of the driven patch alone is shown in Fig. 2.
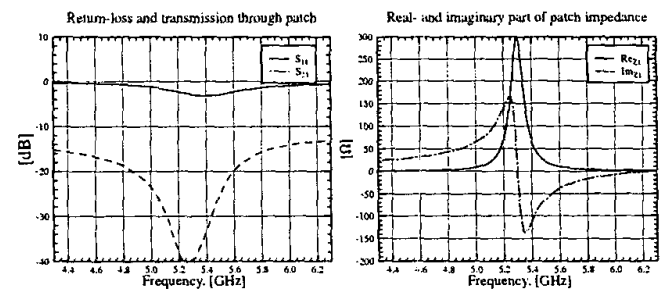

Fig. 2. Measured S-parameters and input impedance of singlelayer C-band microstrip patch.

There is only one resonance in the band shown (approx. at $5.3 \mathrm{GHz}$ ). The impedance for the two ports is identical. An interesting experimental observation is, that the resonance frequency occurs at the same frequency where the two ports are best de-coupled (i.e. at the frequency where the scattering parameter $S_{3}$ exhibits its minimum). At this frequency it can therefore be expected, that the crosspolarization level of the patch will be best, because $S_{\text {, }}$ is minimum. When designing microstrip patches for dualpolarization purposes, it is important to obtain a low level of the element $S_{1}$, since the "cross-coupled" power will directly affect the cross-polarization level.

The impedance match of the single-layer patch to $50 \Omega$ (i.e. the scattering parameter $S_{11}$ ) is quite poor, but this is a direct consequence of the patch being fed at the edge. Here the input impedance is on the order of $300 \Omega$. If a single-layer patch is desired to have a good intrinsic match to a lower impedance level (e.g. $50 \Omega$ ), this can easily be accomplished simply by moving the feed points towards the patch center.

The measured radiation pattern of the single-layer probe-fed microstrip patch is shown in Fig. 3. All pattern measurements presented in this paper have been performed in the spherical near-field test facility of EMI, [8].
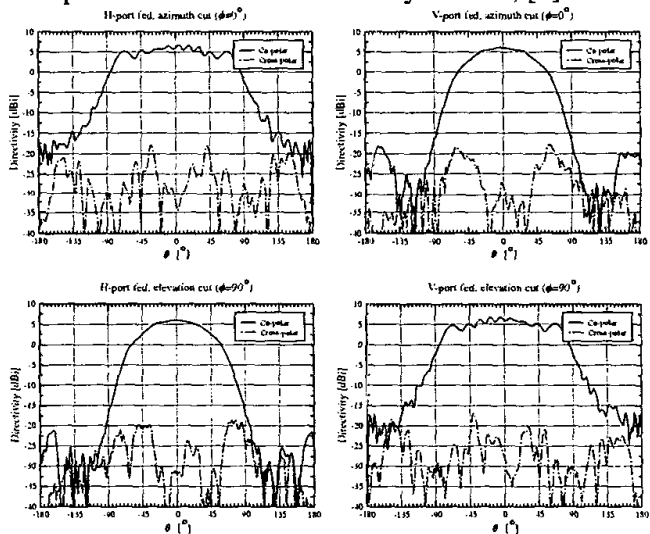

Fig. 3. Radiation pattern of single (unstacked) microstrip patch $(\mathrm{f}=5.3 \mathrm{GHz}$ )

The co-polarized fields in the E-planes (i.e. H-port fed azimuth cut and V-port fed, elevation cut) show the typical rippled behaviour due to diffraction at the edges of the finite sized $(0.5 \mathrm{~m} \times 0.5 \mathrm{~m})$ ground plane. The co-polarized fields in the patch's H-plane, on the other hand, have a much smoother appearance. The cross-polarization level in the main beam is seen to be approximately of the same magnitude as the level of the transmission through the patch (approx. $-30 \mathrm{~dB}$ at $5.3 \mathrm{GHz}$ which is almost the same as the "intrinsic element- $S_{21}$ ", i.e. $S_{21}$ measured, but corrected for mismatch loss). The peak directivity is approx. $6 \mathrm{dBi}$.

As shown in Fig. 2 a single-layer patch can far from achieve the $800 \mathrm{MHz}$ bandwidth required, and a stacked patch is therefore added. This configuration is shown in Fig. 4. 



b)

Fig. 4. Dual polarization probe-fed microstrip stacked patch; a) Top view of the lower patch, b) cross section.

The driven patch is identical to the one shown in Fig. 1. The upper patch acts as a passive parasitic element. Although the impedance of the single (i.e. unstacked) driven patch, when fed on its edge, is very high (see Fig. 2), it is significantly reduced when being loaded by the stacked patch. Hence, to obtain an approx. $50 \mathrm{Ohm}$ input impedance of the stacked patch, the driven patch is fed on the edges. The dual linear polarization requirement implies, that the stacked microstrip patch must be constructed symmetrically. A pair of quadratic and co-axially aligned patches is therefore used.

Since no direct synthesis method for the design of wideband stacked microstrip patches is known, an iterative numerical design process, using an electromagnetic simulator, was adopted. The sizes of and the distance between the two patches were varied, until the desired wideband performance was obtained. During this process it was found, that a good starting point was to design the driven patch first, having its resonance frequency lying in the centre of the band of interest. The stacked patch adds a second resonance, and the task is now to find the parameters so that the two resonances balance in value (i.e. are excited equally strong), and are spaced in frequency so, that the impedance remains constant over the band

Using this "numerical iterative design", combined with impedance measurements to validate the approach, a stacked C-band microstrip patch yielding an near-optimum result was found, when the size of the guadratic lower and upper patches were $14.5 \mathrm{~mm}$ and $19.5 \mathrm{~mm}$, respectively. The upper patch is etched on a $0.1 \mathrm{~mm}$ FR4 substrate, and mounted inverted to let the FR4 material act as environmental protection. The spacing between the patches is $4.5 \mathrm{~mm}$, using a Rohacell $31 \mathrm{HF}$ foam material having $\varepsilon$ of 1.05 . All layers are glued together, using a $0.1 \mathrm{~mm}$ thick adhesive film (supplied by 3M; type 665). The measured scattering parameters for this element are shown in Fig. 5.

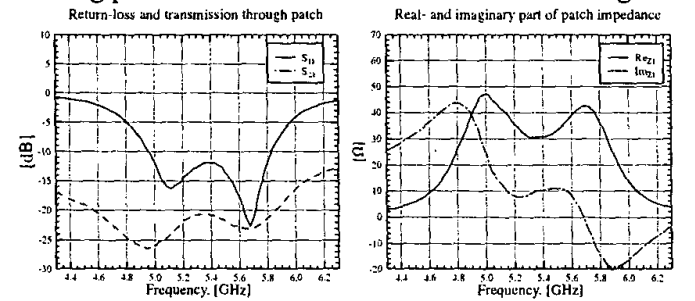

Fig. 5 Measured S-parameters and input impedance of wideband, stacked $\mathrm{C}$-band microstrip patch.

A wideband behaviour for the stacked patch is indeed observed. Note, that the two "peaks" of the real-part input impedance balance quite well. Also, note how the parasitic loading of the lower patch leads to a significant decrease of the input impedance, compared to the single-layer patch. It is seen, that the intrinsic impedance of the stacked patch is not very different from $50 \Omega$ (S of the unmatched patch remains below $-10 \mathrm{~dB}$ in a $50 \Omega$ system, over a $860 \mathrm{MHz}$ bandwidth). This typical characteristic of the stacked patches eases the matching of the element to $50 \Omega$.

Rather than matching the element to $50 \Omega$, it should be considered to design the entire BFN using a slightly lower reference impedance (e.g. $40 \Omega$ ). This will only require one transformation from $40 \Omega$ to $50 \Omega$ (at the input of the array), thus saving board space and reducing the losses (since the loss in a $40 \Omega$ system will be lower than the loss in a $50 \Omega$ system). If reducing the system impedance to $40 \Omega$ and implementing a simple microstrip matching network at the patch feeding point, this has been found to bring $S_{1}$ of the patch down below $-20 \mathrm{~dB}$, over the $800 \mathrm{MHz}$ bandwidth.

Note that the transmission through the patch (i.e. the scattering parameter $S_{2}$ ) is seen to exhibit a similar "stagger-tuned" characteristic as seen in the real-part of the input impedance. The average level of $S_{2}$ for the stacked patch is somewhat poorer than for the single-layer patch, but from a network point-of-view this stagger-tuned behaviour is not surprising. Due to this characteristic of $S_{2}$ it must be expected, that the cross-polarization of the stacked patch is slightly worse, than for the single-layer patch. The "staggertuned" behaviour of $S$ of a stacked patch may probably be the reason why stacked patches are sometimes claimed in the literature to have a poorer cross-polarization performance, than single-layer patches. The above level of $S_{2,}$ (between $-20 \mathrm{~dB}$ and $-25 \mathrm{~dB}$ ) is typical for wideband, probe-fed stacked microstrip patches.

The measured radiation pattern of the stacked microstrip patch is shown in Fig. 6 .
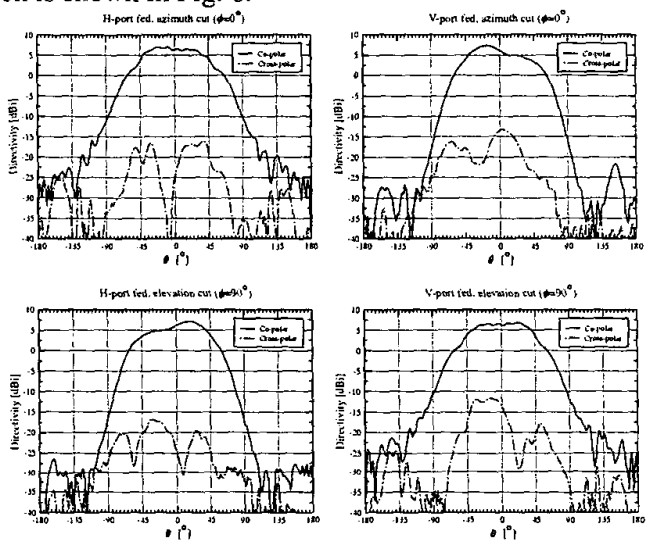

Fig. 6. Measured radiation pattern of stacked microstrip patch $(f=5.3 \mathrm{GHz})$.

The stacked patch radiation pattern has a slightly higher directivity, compared to the unstacked patch. The H-plane pattern of the stacked patch is somewhat asymmetrical (elevation-plane for the H-port fed; azimuth plane for the Vport fed). This behaviour is neither expected, nor understood, and is believed to have been caused by either improper alignment of the two patches or by passive loading of the driven patch by adjacent (non-driven, but stifl mutually coupled) patches. A new measurement is planned to investigate this further, and will be reported at the conference (previously stacked patches designed at EMI have all had well-behaved patterns). Despite this slight pattern anomaly, the co- and cross-polarization patterns are both quite usable for arrays. The influence of the ground plane is much less significant, than for the single-layer patch. The pattern remain stable over the full $800 \mathrm{MHz}$ bandwidth, but the main beam narrows with increasing frequency, and the associated peak directivity increases from $6.5 \mathrm{dBi}$ at $5.1 \mathrm{GHz}$ to $10 \mathrm{dBi}$ at $5.9 \mathrm{GHz}$.

The cross-polarization level for the stacked patch is worse than for the single-layer patch, but this was expected, due to its higher $S_{21}$-level. If this level of cross-polarization is not compliant with array requirements, simple, yet very efficient, techniques can be employed in the large array to substantially improve the overall array cross-polarization suppression over that of the basic stacked patch element. A cross-polarization improvement of typical $15 \mathrm{~dB}$ in microstrip patch arrays has been achieved using the method described in [9] and [10]. A practical example of the implementation of this cross-polarization improvement technique is found in [7] 
To investigate wideband array-issues, four stacked patch elements were combined into a $2 \times 2$ element group, as shown in Fig. 7. The S-parameters of the array was measured in the case where the elements were connected to two four-way Wilkinson power splitters/combiners using coaxial cables (i.e. all elements were fed in equal amplitude and phase). The element spacing was approx. $0.7 \lambda_{0}$.

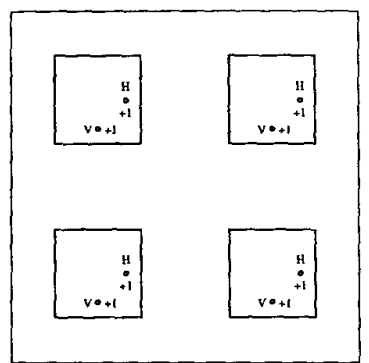

Fig. 7. Layout of $2 \times 2$ element stacked microstrip array.

The measured S-parameters are shown in Fig. 8, and are compared with the calculated S-parameters in the two cases: a) Mutual coupling between the elements is neglected in the calculation, b) All mutual couplings between the elements are taken into account. In the a) case only the four eigenimpedances of the patches were connected together (in the computer) with the measured S-parameters of the four-way Wilkinson power splitters and associated cables. In the b) case, all S-parameters of the $2 \times 2$ element dual-polarized array (i.e. the full eight-port), all cables, and the power dividers were measured and connected.
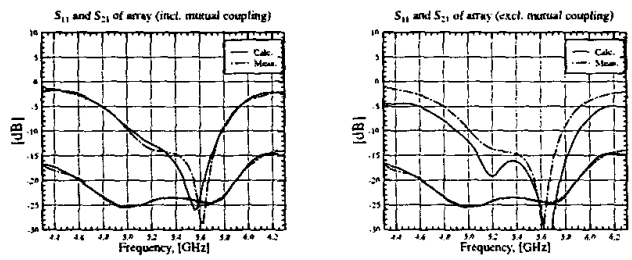

Fig. 8. Measured S-parameters of the $2 \times 2$ element stacked Cband microstrip array

From Fig. 8 it is observed, that the bandwidth of the $2 \times 2$ element group is slightly lower than for the individual stacked element. This is due to mutual coupling between the elements, and to a lesser extent due to the finite bandwidth of the Wilkinson divider. Note that $S_{1}$ of the array is practically identical to $S_{2}$ for the element. Also note, that the mutual coupling certainly must be taken properly into account, otherwise measured vs. calculated data does not agree very well. The measured radiation pattern of the $2 \times 2$ element array is shown in Fig. 9.


Fig. 9. Radiation pattern of $2 \times 2$ element array of stacked microstrip patches $(f=5.3 \mathrm{GHz}$, uniform excitation).
The radiation pattern is symmetrical, does not show any sign of anomaly, and has deep nulls. The peak directivity is $13.9 \mathrm{dBi}$ at $5.3 \mathrm{GHz}$, increasing to $14.8 \mathrm{dBi}$ at $5.9 \mathrm{GHz}$. The cross-polarization level is on the order of $-25 \mathrm{~dB}$, which is slightly better than for the individual element. Measurements confirm that the pattern is stable over a very wide frequency range (in excess of $1 \mathrm{GHz}$ ), and that the cross-polarization level remains low.

As demonstrated, low-cost stacked microstrip probe-fer patch arrays can be designed, capable of operating over a 15 $\%$ bandwidth in $\mathrm{C}$-band. What still remains in order to arrive at a complete antenna, is the design of the azimuth and elevation beam forming networks.

\section{Future work}

The C-band element described here is presently being integrated into a full dual-frequency array ( $\mathrm{L}$ - and C-band). This array will be based on the use of perforated, stacked $\mathrm{L}$ band patches [11], inside the perforations of which the Cband elements are placed.

\section{Conclusion}

The design and performance of a wideband, dualpolarization probe-fed microstrip C-band antenna element and a small test array, have been described. The element will be used in an $800 \mathrm{MHz}$ dual-polarization array, being designed for use with the next-generation of the Danish airborne SAR system, EMISAR. The dual-polarization probe-fed microstrip array described here is believed to be the most widebanded antenna of its kind yet reported, for use in polarimetric SAR systems.

\section{Acknowledgements}

This work was supported by the Danish National Research Foundation.

\section{References}

[1] JPL's "LightSAR", see e.g. http://lightsar.jpl.nasa.gov/

[2] European Space Agency's "Envisat", see e.g. 3] Canadian Space Agency's "RADARSAT Il", see e.g http://www.space.gc.ca/eng/about/radarsat/rad8.html

[4] Department of Electromagnetic System, Technical University of Denmark's next-generation SAR, "SAR++". see http://sarppsrv.emi.dtu.dkJ -sarpp/

[5] E.L. Christensen, N. Skou, J. Dall, K. Woelders, J.H førgensen, J. Granholm, S.N. Madsen, "EMISAR: An absolutely calibrated polarimetric $L$ - and C-band SAR", IEEE Trans. on Geoscience and Remote Sensing Applications, Vol. 36, no. 6, Nov 1998, pp. 1852-1865

[6] J. Granholm, K. Woelders, M. Dich, E. Lintz Christensen "Microstrip antenna for polarimetric C-band SAR", IEEE Int Symp. on Antennas and Propagation, Seattle, WA, June 1994, pp $1844-1847$

[7] J. Granholm, K. Woelders, "Dual polarization microstrip antenna array with very low cross-polarization", submitted for publication in IEEE Trans. on Antennas and Propagation.

[8] J.E. Hansen, F. Jensen, "Spherical near-field scanning at the Technical University of Denmark", IEEE Trans. on Antennas and Propagation., Vol. 36, June 1988, pp. 734-739.

[9] J. Granholm, K. Woelders, "Dual-polarization antenna array with very low cross-polarization and low side lobes", International $P C$

[10] K. Woelders, J. Granholm, "Cross-polarization and sidelobe suppression in dual linear polarization antenna arrays", IEEE Trans. on Antennas and Propagation, Vol. 45, No. 12, Dec. 1997, pp. $1727-1740$.

[11] J. Granholm, "Dual-frequency and polarization antenna arrays for future SAR systems", $2 I^{\prime \prime}$ ESTEC Antenna Workshop on Noordwijk, The Netherlands, 6-8 May 1998, pp. 29-35. 